\title{
Epigenetic Approach to PTSD: In the Aspects of Rat Models
}

\author{
Asli Aykac ${ }^{1}$ Rasime Kalkan 2,3 \\ ${ }^{1}$ Department of Biophysics, Faculty of Medicine, Near East University, \\ Nicosia, Cyprus \\ 2 Department of Molecular Biology and Genetics, Faculty of Arts and \\ Sciences, Near East University, Nicosia, Cyprus \\ ${ }^{3}$ Department of Medical Genetics, Faculty of Medicine, Near East \\ University, Nicosia, Cyprus
}

Glob Med Genet 2022;9:7-13.

\author{
Address for correspondence Asli Aykac, PhD, Department of \\ Biophysics, Faculty of Medicine, Near East University Boulevard, Near \\ East University, Nicosia 99138, Cyprus \\ (e-mail: aykacasli@yahoo.com).
}

\begin{abstract}
Keywords

- PTSD

- epigenetics

- methylation

- rat studies

Posttraumatic stress disorder (PTSD) is a stress-related mental disorder and develops after exposure to life-threatening traumatic experiences. The risk factors of PTSD included genetic factors; alterations in hypothalamic-pituitary-adrenal (HPA) axis; neurotrophic, serotonergic, dopaminergic, and catecholaminergic systems; and a variety of environmental factors, such as war, accident, natural disaster, pandemic, physical, or sexual abuse, that cause stress or trauma in individuals. To be able to understand the molecular background of PTSD, rodent animal models are widely used by researchers. When looking for a solution for PTSD, it is important to consider preexisting genetic risk factors and physiological, molecular, and biochemical processes caused by trauma that may cause susceptibility to this disorder. In studies, it is reported that epigenetic mechanisms play important roles in the biological response affected by environmental factors, as well as the task of programming cell identity. In this article, we provided an overview of the role of epigenetic modifications in understanding the biology of PTSD. We also summarized the data from animal studies and their importance during the investigation of PTSD. This study shed light on the epigenetic background of stress and PTSD.
\end{abstract}

\section{Introduction}

Posttraumatic stress disorder (PTSD), which is among the stress-related mental disorders, develops after exposure to life-threatening traumatic experiences. The main risk factor of PTSD is genetic factors which are affected by many important biological systems, such as the hypothalamicpituitary-adrenal (HPA) axis; neurotrophic, serotonergic, dopaminergic, and catecholaminergic systems; and a variety of environmental factors, such as war, accident, natural disaster, pandemic, physical or sexual abuse, that cause stress or trauma in individual. ${ }^{1-3}$

received

August 11, 2021

accepted after revision

September 18, 2021

published online

November 11, 2021
Exposure to trauma varies from person to person, depending on many interacting risk factors, including gender, genetics, and social interaction, and early life experiences. ${ }^{4,5}$ As a result of this difference, the individual may not develop PTSD, or the symptoms seen in people who develop PTSD may spontaneously improve or, on the contrary, the individual may be at high risk of recurrence of symptoms, even though they have recovered from PTSD. ${ }^{6}$ The fact that some individuals are still in the high-risk group for PTSD despite the decrease in symptoms emphasizes that the effects of long-term processes should be taken into account in the response to trauma. Therefore, when seeking a solution for

(c) 2021. The Author(s).

This is an open access article published by Thieme under the terms of the Creative Commons Attribution License, permitting unrestricted use, distribution, and reproduction so long as the original work is properly cited. (https://creativecommons.org/licenses/by/4.0/)

Georg Thieme Verlag KG, Rüdigerstraße 14, 70469 Stuttgart, Germany 
PTSD, it is important to consider preexisting genetic risk factors and physiological, molecular, and biochemical processes caused by trauma that may cause susceptibility to this disorder. Although it is an effective method to use patients while investigating human diseases, it is not ethically appropriate to induce PTSD in healthy individuals and the occurrence of mental disorders such as PTSD in humans is not always real-time but often coincidental. ${ }^{7}$ Since of all these cases that limit the use of human subjects, rodent animal models are generally accepted and used by research groups in PTSD studies.

\section{Existing Rodent Models of Inducing Symptoms Seen in People with PTSD}

Present animal models fall into three different categories to induce symptoms seen in people with PTSD; it uses physical, social, and psychological stress factors, and these stress factors can reveal common or differentiated neuroadaptation patterns in all models. ${ }^{8}$ In physical stress administrations, different stressors are used either alone or in combination to evaluate behavioral responses to stress. The time-dependent sensitization model which is based on sensitivity, mainly uses psychological or pharmacological stressors. The common feature of all these experiments is sensitization. A weak stimulus given after a dangerous stimulus causes an increase in sensitivity, although it is less than the intensity of the life-threatening stimulus. Under natural conditions, sensitization is a principal action to threat as it accelerates escape responses and thus may protect the subject from future danger. ${ }^{9}$ Underwater trauma model is based on forced to swim for 1 minute, then held underwater during 30 seconds. Foot shock is a model in which the electric shock (in different intensity and duration) applied to the foot of the animal placed in the apparatus is used as a physical factor. ${ }^{10}$ With the foot shock model, avoidance and anxiety behavior can reproduce some of the main symptoms of PTSD, including hyperarousal, aggression and flashback, and sleep disturbance. ${ }^{10,11}$ Several studies are used auditory or visual stimuli (situational reminder [SR]) in addition to the classical fear conditioning procedure such as foot shock, using these stimuli given with shock to animals to remember fear aftershock. Unlike the classical model, in the Stress-Enhanced Fear Learning (SELF) model, animals are either not exposed to any shock or are exposed to unpredictable foot shocks many times in a single session. ${ }^{12}$ One of the most widely used PTSD models is the single prolonged stress (SPS) model. In this model, rats are exposed to varying stress factors. In the general SPS protocol, the animal is kept in a restriction cage for 2 hours, then subjected to forced swimming and exposed to diethyl ether until unconscious. ${ }^{13}$ The restraint/immobilization stress model, also used as part of the SPS procedure, is applied by restraining the animal using plastic retention tubes or immobilizing the four limbs and head on a board in a prone position. ${ }^{14}$ It is also possible to benefit from the natural social behaviors of animals with social stress factors, instead of directly stressful stimulants. Since humans are known to develop PTSD in response to social experiences, such as rape and childhood abuse, it is reasonable to think that this applies to another species as well.

PTSD models which are using social stressors are often used in conjunction with other PTSD psychological models. The housing instability (HI) model is applied by placing the animal randomly in cages with different animals each time. $^{15}$ This model makes sense considering that PTSD patients are affected by HI. ${ }^{16}$ Animals subjected to this model are generally exposed to the psychosocial stress model at first. As a psychosocial stress model, predatorbased models are generally used. The predator-based model is based on the hypothesis that a synergistic effect develops due to the interaction of risk factors and PTSD. Applied at different times and it includes exposure to predator stressor, random cage change, and immobilization stress application procedures. ${ }^{15,17}$ In predator-based models, animals are exposed to either the predator itself or a clue of the predator, such as urine, faces, or feathers. Studies have shown that rats have a strong innate fear of a predator. ${ }^{18}$ There are many studies in the literature in which trimethylthiazolin (TMT), a synthetic compound isolated from other predator odors and urine, is used instead of living animals. ${ }^{19}$ The Predator Scent Stress is the model in which animals are exposed to the scent of their natural predator to model how humans reproduce variations in response to trauma. For example, stress group rats are exposed to dirty contaminated cat litter for 10 minutes, while rats in the control group are exposed only to clean litter for the same period. On the last day when the behavioral parameters of the animals will be evaluated with a maze (elevated plus, $\mathrm{T}$ or radial) or/and open field test mechanisms, animals in all groups (both stress and control groups) are exposed to clean cat litter for 10 minutes, creating a situation that reminds trauma and reexperiencing trauma. ${ }^{20}$

Recall of traumatic memories, which are among the symptoms of PTSD, is directly related to dysregulation and deterioration in the reconstruction of fear memory. ${ }^{21}$ Moreover, traumatic events can also affect memory formation as they often marked strong memories. Therefore, PTSD models which using fear conditioning is focused on the processing of fear memory, behavioral responses, physiological, molecular, and neuro epigenetic processes in the basic brain regions responsible for fear learning and memory processes such as the hippocampus, amygdala, and frontal cortex. ${ }^{21-23}$

After different combinations of procedures, animals display impaired habituation behaviors in their new environment. ${ }^{24}$ It is also possible to induce PTSD-like symptoms using social isolation and maternal separation (early life stress model) such as the random cage change made in the HI model. ${ }^{25,26}$ In the social defeat-SD model, which is used to trigger-off avoidance behavior among PTSD symptoms, subjects are exposed to a single aggressive animal. ${ }^{27,28}$ Although both physical and social stress factors produce behavioral and neuroendocrine changes observed in PTSD, most of the relevant models do not take into account that people show different responses to trauma, some individuals are sensitive to PTSD, and some are resistant. Psychological stress, which 
elicits instinctive responses in animals to natural predators, more successfully reveals the difference between those susceptible to the development of PTSD and resistant ones. ${ }^{7}$

The models that follow the provocation of fear and stress in animals are based on the elimination of the stress factor and the comparison of recovery and related biological responses with homeostasis. Animal models of PTSD can mimic human symptoms quite well, depending on the conditioning or triggering stressor factor. Unfortunately, animal models cannot explain well the differences between individuals in fear acquisition and disappearance of fear. ${ }^{29,30}$

PTSD models that expose animals to the factors of trauma before fear conditioning may be said to have greater validity than fear conditioning alone. To be able to determine the epigenetic effect of trauma and stress subsequently, fearlearning stress and/or trauma-enhanced fear learning models has been used. These studies have been provided to define the important role of drain-derived neurotrophic factor (BDNF) on stress and fear memory. According to the results of the SEFL paradigm study, the first response is included the hippocampal BDNF/TrkB signal to fear to condition which increases exon I and IV BDNF mRNA levels, acetylation in H3 and $\mathrm{H} 4$ histones, and TrkB protein levels in the exon I and IV BDNF promoter regions. BDNF, which is considered to be a key regulator of the development of neurons in the central nervous system, is thought to be an important factor mediating synaptic plasticity. ${ }^{31,32}$

There is accumulating evidence in the literature that traumatic experiences are associated with epigenetic modifications in the human genome. ${ }^{33}$ Epigenetic modifications are associated with alteration in gene expression. Also, the epigenome is one of the susceptibility factors which contributes to PTSD. ${ }^{33,34}$

\section{Epigenetic Alterations in Posttraumatic Stress Disorder}

Genetic and environmental factors play a key role during the development of PTSD. Advancement of molecular and genetic diagnostic methods help identification of genetic alterations of PTSD. ${ }^{34}$ Studies highlighted that parental exposure to posttraumatic stress can cause potential biological alterations in their offspring. ${ }^{35,36}$ These studies show the importance of the heritability of PTSD and paved the way for genetic studies. Clinical diagnosis of PTSD is based on behavioral symptom clusters that are most directly associated with brain function, epigenetic studies of PTSD in humans have been limited to peripheral tissues such as blood, buccal tissue, and saliva. Animal models of PTSD, predominantly in rodents, have been used for the identification of the epigenetic alterations in the brain.

Epigenetic modifications are effects on gene expression status without altering nucleotide sequence. Epigenetic modifications involve DNA methylation, histone modifications, RNA modifications, and noncoding RNAs. Epigenetic alterations are influenced by both environmental and genetic factors. Epigenetic alterations are under control by several enzymes, like DNA methyltransferases, DNA demethylases, lysine methyltransferases and demethylases, and so on. The reversible nature of the epigenetic alterations is the crucial point of these modifications, and this makes them a potential target during the evaluation of disease and as a target for the treatment planning.

\section{Histone Modification}

Histone proteins are important during the packaging of DNA and directly related to gene regulation. Histones chemically modified through the action of enzymes to regulate gene transcription. Histone acetyltransferase (HAT) is responsible for histone acetylation and acetylation of histones causes the noncondensing or loosening of DNA which allows transcription. Histone deacetylase (HDAC) is responsible to remove the acetyl group from histones and causes the coiling of DNA, creating condensed densely packed DNA which inhibits or repress the gene expression. Histone modifications are attached N-terminal tail of histones covalently. These modifications are involved acetylation, methylation, phosphorylation, ubiquitination, and sumoylation. ${ }^{37}$ Lubin and Sweatt demonstrated that a global increase in histone $\mathrm{H} 3$ acetylation and phosphorylation was related to recalling of fear memory in the CA1-4 region of the hippocampus ${ }^{38}$ and Maddox and Schafe showed recalling fear memory promoted increased level acetylation of histone $\mathrm{H} 3$ in the lateral amygdala. ${ }^{39}$ In a study using a rodent model of PTSD, it was shown that histone hyperacetylation was triggered in LA after ingestion of fear memory inhibitors. ${ }^{40}$

Studies demonstrated strong interaction between fear and histone acetylation. ${ }^{41,42}$ Webb et al demonstrated the interaction between fear memory and H3K4me3 and 5hydroxymethylcytosine (5-hmc) in the hippocampal CA1 region and anterior cingulate cortex (ACC). ${ }^{42}$ Therefore, these studies show us the interaction between epigenetic alterations and their effects on different brain region during fear memory formation. Takei et al used an SEFL paradigm with an SPS in rats and observed an increased level of acetylation of histone $\mathrm{H} 3$ and $\mathrm{H} 4$ at the exon I and IV BDNF promoter regions in SPS rats in their hippocampus. ${ }^{43}$ Takei et al demonstrated enhanced hippocampal BDNF/TrkB signaling in response to fear conditioning in this animal model of PTSD. $^{43}$ The reversible nature of epigenetic modifications makes them a therapeutic target. HDAC, DNA Methyltransferases (DNMT), and Histone methyltransferases (HMT) inhibitors are widely used inhibitors in clinical practice and researches which changes the effects of modifications especially in cancer. Studies emphasized that drugs targeting HDACs and DNA methyltransferase inhibitors as an emerging anticancer strategy and The Food and Drug Administration (FDA) has approved these agents as a treatment target. Lee et al demonstrated that knockdown of HDAC6 prevents the enhancement of glutamatergic signaling by acute stress in rats. ${ }^{44}$ Studies demonstrated that HDAC inhibitors sodium butyrate $(\mathrm{NaB})$, trichostatin $\mathrm{A}$, and valproic acid which elevate histone acetylation levels, has therapeutic effects in rodent stress paradigms, improving cognition, and reducing anxiety behaviors. ${ }^{45}$ 


\section{DNA Methylation}

DNA methylation is one of the most studied epigenetic alterations. Methyl groups bind covalently fifth carbon of cytosine nucleotides on DNA. DNA methylation is a reversible process and DNA methyltransferases are responsible for adding methyl groups and DNA demethylases are responsible for removing those methyl groups. Methylation of cytosine nucleotide is directly related to gene expression. DNA methylation is important during tissue differentiation, tissue-specific gene expression, and $\mathrm{X}$ inactivation. ${ }^{46}$ Advancement of diagnostic tools will help identification of different DNA modifications, like 5-methylcytosine $(5-\mathrm{mc})$ and 5hmc. ${ }^{37,47}$ These modifications are important in PTSD and learning.

Methylation studies showed that FK506 binding protein 51 gene, BRSK1, LCN8, NFG, DOCK2, ZFP57, RNF39, ${ }^{46-48}$ BDNF, NR3C1, MAN2C1, TLR8, SLC6A4, IL-18, SKA2, ${ }^{3}$ LINC01090, BC036345, ZNRD1-ASI, and RORA, ${ }^{49-55}$ TPR, ANXA2, CLEC9A, ACP5, TLR8, LRRC3B, BRSK1, LCN8, NGF, DOCK2 ${ }^{48}$ DUSP22, HIST1H2APS2, HOOK2, NINJ2, PAX8, RNF39, ZFP57, ${ }^{56}$ NRG1, HGS genes are related with PTSD and severity of symptoms ${ }^{48,56,57}$ (-Table $\mathbf{1}$ ). The FK506-binding protein 51 (FKBP5) gene is one of the genes on HPA axis and an important regulator of stress response through altering glucocorticoid receptor sensitivity. ${ }^{58}$ Klengel et al identified an interaction between demethylation of FKBP5 and early trauma exposure..$^{55}$ Rodent studies demonstrated that parental care influences revealed that differences in maternal phenotypes effects their pup's development of behavioral and HPA responses to stress as adults. ${ }^{59}$ Elevated level DNA methylation was identified in the offspring after low maternal care and lower methylation was identified in high maternal care. ${ }^{59,60} \mathrm{Li}$ et al demonstrated the interaction between fear extinction and Tet3-mediated global 5-hmc in the infralimbic region of the ventromedial prefrontal cortex. $^{61}$

Studies highlighted that different brain regions, like dentate gyrus (DG), dorsal DG, dorsal CA1, ventral CA3, basolateral amygdala, and medial prefrontal cortex, show different level of gene expression during the stress induction. ${ }^{62}$ Roth et al reported that in PTSD, BDNF exon IV was hypomethylated in the ventral CA3 and no methylation changes observed in other hippocampal subregions in rats. ${ }^{63}$ It is also reported in the literature that there is a relationship between the epigenetic status of catechol-Omethyltransferase and PTSD. ${ }^{64}$

$N R 3 C 1$ is another most studied gene which encodes the GR. NR3C1 plays a key role in the understanding of the epigenetic regulation of PTSD and fear extinction processes. ${ }^{65}$ As a result of the study examining the relationship between the methylation level of the NR3C1 promoter and PTSD, it is emphasized that the higher level of DNA methylation in the NR3C1 promoter region could be a protective effect against vulnerability to PTSD. ${ }^{66}$

HDAC4 gene was related to learning and memory-related processes and studies demonstrated that higher level

Table 1 Differentially methylated human genes in PTSD

\begin{tabular}{|l|l|l|l|l|}
\hline Gene & Tissue type & Species & Methylation status & Reference \\
\hline BRSK1 & Blood & Human & Hyper & $46-48$ \\
\hline LCN8 & Blood & Human & Hyper & $46-48$ \\
\hline NFG & Blood & Human & Hyper & $46-48$ \\
\hline DOCK2 & Blood & Human & Hyper & $46-48$ \\
\hline ZFP57 & Blood & Human & Hyper & $46-48$ \\
\hline RNF39 & Blood & Human & Hyper & $46-48$ \\
\hline NR3C1 & Whole blood & Human & Hypo & 3 \\
\hline MAN2C1 & Whole blood & Human & Hypo & 3 \\
\hline TLR8 & Whole blood & Human & Hypo & 3 \\
\hline SLC6A4 & Whole blood & Human & Hypo & 3 \\
\hline IL-18 & Whole blood & Human & Hypo & 3 \\
\hline SKA2 & Whole blood & Human & Hypo & 3 \\
\hline DUSP22 & Blood & Human & NS & 49 \\
\hline HIST1H2APS2 & Blood & Human & Hypo & 49 \\
\hline HOOK2 & Blood & Human & NS & 49 \\
\hline NIN12 & Blood & Human & NS & 49 \\
\hline PAX8 & Blood & Human & NS & 49 \\
\hline RNF39 & Blood & Human & Hypo & 49 \\
\hline ZFP57 & Blood & Hyman & 49 \\
\hline
\end{tabular}

Abbreviations: Hyper, hypermethylation in case relative to control; Hypo, hypomethylation in case relative to control; NS, nonspecified; PTSD, posttraumatic stress disorder. 
methylation was observed in PTSD cases. ${ }^{63}$ In the study where BDNF, NR3C1, MAN2C1, TLR8, SLC6A4, IL-18, and SKA2 gene methylation analyses were performed in patients suffering from PTSD, it was concluded that BDNF, NR3C1 and MAN2C1 methylations are associated with PTSD diagnosis. ${ }^{3}$ This study revealed the importance the regulation of genes involved in synaptic plasticity and the HPA axis and their association with PTSD.

\section{Noncoding RNA}

Noncoding RNAs (ncRNAs) are not translated into a polypeptide. They play a key role during the processing and regulation of other RNAs such as messenger RNA (mRNA), transfer RNA (tRNA), and ribosomal RNA (rRNA). ${ }^{67,68}$ Recent studies showed the importance of ncRNAs as a biomarker for trauma-related brain disorders. ${ }^{69}$ Ambeskovic et al demonstrated how transgenerational and multigenerational stress factors regulate cortical miR-221 and miR-26 expression and their target genes, ntrk2, and map1a, and crh in the rat. They concluded that early life experiences and prenatal stress are crucial factors for brain development and mental health. ${ }^{70}$

Maurel et al studied miR-15a-5p, miR-497a-5p, miR-511$5 p$, and let-7d-5p in brain areas of mouse model of PTSD. ${ }^{71}$ They identified lower transcript levels of miR-15a-5p, miR497a-5p, and miR-511a-5p in the hippocampus and hypothalamus and in the medial prefrontal cortex, downregulation of miR-15a-5p, miR-511-5p, and let-7d-5p. They concluded that miRNA expression in the different brain areas correlated to miRNA-based epigenetic modulation in stressinduced phenotypes. ${ }^{71}$

Li et al demonstrated that exposure of acute traumatic stress in early adolescent caused permanent changes in neural network and altered expression of CRFR1 and CRFR1 mRNA and miR-34c expression in Wistar rats. ${ }^{72}$ These studied showed the importance of identification novel non coding RNAs as a candidate for biomarkers of PTSD.

\section{Conclusion}

PTSD characterized by systemic dysregulation in anatomical sites outside of the brain, maladaptive alterations of the HPA axis, and sympathetic nervous system sensitivity/responsivity, and alterations in neuroimmune dynamics. ${ }^{59}$ Human-based epigenetic studies of PTSD demonstrated alterations in agerelated CpGs, DNA methylation, alteration in estrogen-responsive genes, alterations in the dopaminergic system, and alterations in the HPA axis. Environmental factors may have longterm consequences on brain behavior as a result of interaction with the genome by regulating epigenetic mechanisms. The susceptibility of genes due to stress and/or trauma, the effect of epigenetic changes on the development of PTSD has recently become more remarkable. In the literature, methylation analyses were performed using genes encoding proteins, such as BDNF, FKBP5, IL-18, MAN2C1, NR3C1, SKA2, SLC6A4 and TLR8, which play a role in the synaptic plasticity, immune system, serotonin modulation, neurogenesis, and inflammation response in PTSD., ${ }^{3,73}$ While clinical researches are vital in the administration of new therapeutic agents to people with PTSD, animal models are indispensable for comparing the behavioral and physiological effects caused by trauma, as well as molecular and biochemical abnormalities and the state of homeostasis.

Epigenetics studies provide a crucial role in understanding the interaction of epigenetics with environmental exposure to trauma in PTSD. Further studies will shed light on the interaction between stress-induced epigenetic regulations of neuronal function. The reversible nature of epigenetic modifications promises for determination of the epigenetic architecture of PTSD and may play a crucial role during the discovery of novel targeted therapeutic approaches to develop treatment strategies and prevention of PTSD. These studies will shed light on resolving several issues, like epigenetic treatment, trauma, or stress memory. Studies in the field of epigenetics will help to discover a marker for susceptibility to PTSD.

Funding

None.

Conflict of Interest

None declared.

\section{References}

1 Gradus JL, Qin P, Lincoln AK, et al. Posttraumatic stress disorder and completed suicide. Am J Epidemiol 2010;171(06): 721-727

2 Forte G, Favieri F, Tambelli R, Casagrande M. COVID-19 pandemic in the Italian population: validation of a post-traumatic stress disorder questionnaire and prevalence of PTSD symptomatology. Int J Environ Res Public Health 2020;17(11):4151-4164

3 Hossack MR, Reid MW, Aden JK, Gibbons T, Noe JC, Willis AM. Adverse childhood experience, genes, and PTSD risk in soldiers: a methylation study. Mil Med 2020;185(3-4):377-384

4 Zoladz PR, Diamond DM. Predator-based psychosocial stress animal model of PTSD: Preclinical assessment of traumatic stress at cognitive, hormonal, pharmacological, cardiovascular and epigenetic levels of analysis. Exp Neurol 2016;284(pt. B):211-219

5 Voisey J, Young RM, Lawford BR, Morris CP. Progress towards understanding the genetics of posttraumatic stress disorder. J Anxiety Disord 2014;28(08):873-883

6 Solomon Z, Mikulincer M. Trajectories of PTSD: a 20-year longitudinal study. Am J Psychiatry 2006;163(04):659-666

7 Borghans B, Homberg JR. Animal models for posttraumatic stress disorder: An overview of what is used in research. World J Psychiatry 2015;5(04):387-396

8 Warren BL, Vialou VF, Iñiguez SD, et al. Neurobiological sequelae of witnessing stressful events in adult mice. Biol Psychiatry 2013; 73(01):7-14

9 Antelman SM. Time-dependent sensitization as the cornerstone for a new approach to pharmacotherapy - drugs as foreign stressful stimuli. Drug Dev Res 1988;14:1-30

10 Louvart H, Maccari S, Ducrocq F, Thomas P, Darnaudéry M. Longterm behavioural alterations in female rats after a single intense footshock followed by situational reminders. Psychoneuroendocrinology 2005;30(04):316-324

11 Pawlyk AC, Jha SK, Brennan FX, Morrison AR, Ross RJ. A rodent model of sleep disturbances in posttraumatic stress disorder: the role of context after fear conditioning. Biol Psychiatry 2005;57 (03):268-277 
12 Rau V, Fanselow MS. Exposure to a stressor produces a long lasting enhancement of fear learning in rats. Stress 2009;12(02): 125-133

13 Liberzon I, Krstov M, Young EA. Stress-restress: effects on ACTH and fast feedback. Psychoneuroendocrinology 1997;22(06): 443-453

14 Balkaya M, Prinz V, Custodis F, et al. Stress worsens endothelial function and ischemic stroke via glucocorticoids. Stroke 2011;42 (11):3258-3264

15 Zoladz PR, Conrad CD, Fleshner M, Diamond DM. Acute episodes of predator exposure in conjunction with chronic social instability as an animal model of post-traumatic stress disorder. Stress 2008;11(04):259-281

16 Kim HG, Harrison PA, Godecker AL, Muzyka CN. Posttraumatic stress disorder among women receiving prenatal care at three federally qualified health care centers. Matern Child Health J 2014;18(05):1056-1065

17 Zoladz PR, Fleshner M, Diamond DM. Psychosocial animal model of PTSD produces a long-lasting traumatic memory, an increase in general anxiety and PTSD-like glucocorticoid abnormalities. Psychoneuroendocrinology 2012;37(09):1531-1545

18 Blanchard RJ, Blanchard DC, Rodgers J, Weiss SM. The characterization and modelling of antipredator defensive behavior. Neurosci Biobehav Rev 1990;14(04):463-472

19 Apfelbach R, Blanchard CD, Blanchard RJ, Hayes RA, McGregor IS. The effects of predator odors in mammalian prey species: a review of field and laboratory studies. Neurosci Biobehav Rev 2005;29(08):1123-1144

20 Cohen H, Geva AB, Matar MA, Zohar J, Kaplan Z. Post-traumatic stress behavioural responses in inbred mouse strains: can genetic predisposition explain phenotypic vulnerability? Int J Neuropsychopharmacol 2008;11(03):331-349

21 Careaga MBL, Girardi CEN, Suchecki D. Understanding posttraumatic stress disorder through fear conditioning, extinction and reconsolidation. Neurosci Biobehav Rev 2016;71:48-57

22 Zovkic IB, Sweatt JD. Epigenetic mechanisms in learned fear: implications for PTSD. Neuropsychopharmacology 2013;38(01): 77-93

23 Kwapis JL, Wood MA. Epigenetic mechanisms in fear conditioning: implications for treating post-traumatic stress disorder. Trends Neurosci 2014;37(12):706-720

24 Saavedra-Rodríguez L, Feig LA. Chronic social instability induces anxiety and defective social interactions across generations. Biol Psychiatry 2013;73(01):44-53

25 Imanaka A, Morinobu S, Toki S, Yamawaki S. Importance of early environment in the development of post-traumatic stress disorder-like behaviors. Behav Brain Res 2006;173(01):129-137

26 Cloitre M, Stolbach BC, Herman JL, et al. A developmental approach to complex PTSD: childhood and adult cumulative trauma as predictors of symptom complexity. J Trauma Stress 2009;22 (05):399-408

27 Pulliam JV, Dawaghreh AM, Alema-Mensah E, Plotsky PM. Social defeat stress produces prolonged alterations in acoustic startle and body weight gain in male Long Evans rats. J Psychiatr Res 2010;44(02):106-111

28 Yang R, Daigle BJ Jr., Muhie SY, et al. Core modular blood and brain biomarkers in social defeat mouse model for post traumatic stress disorder. BMC Syst Biol 2013;7:80

29 Ursano RJ, Li H, Zhang L, et al. Models of PTSD and traumatic stress: the importance of research "from bedside to bench to bedside". Prog Brain Res 2008;167:203-215

30 Yehuda R, Hoge CW, McFarlane AC, et al. Post-traumatic stress disorder. Nat Rev Dis Primers 2015;1:15057

31 Frielingsdorf H, Bath KG, Soliman F, Difede J, Casey BJ, Lee FS. Variant brain-derived neurotrophic factor Val66Met endophenotypes: implications for posttraumatic stress disorder. Ann N Y Acad Sci 2010;1208:150-157
32 Matsuoka Y, Nishi D, Noguchi H, Kim Y, Hashimoto K. Longitudinal changes in serum brain-derived neurotrophic factor in accident survivors with posttraumatic stress disorder. Neuropsychobiology 2013;68(01):44-50

33 Maddox SA, Watts CS, Doyère V, Schafe GE. A naturally-occurring histone acetyltransferase inhibitor derived from Garcinia indica impairs newly acquired and reactivated fear memories. PLoS One 2013;8(01):e54463

34 Sartor CE, McCutcheon VV, Pommer NE, et al. Common genetic and environmental contributions to post-traumatic stress disorder and alcohol dependence in young women. Psychol Med 2012; 41(07):1497-1505

35 Leen-Feldner EW, Feldner MT, Knapp A, Bunaciu L, Blumenthal H, Amstadter AB. Offspring psychological and biological correlates of parental posttraumatic stress: review of the literature and research agenda. Clin Psychol Rev 2013;33(08):1106-1133

36 Lambert JE, Holzer J, Hasbun A. Association between parents' PTSD severity and children's psychological distress: a metaanalysis. J Trauma Stress 2014;27(01):9-17

37 Basavarajappa BS, Subbanna S. Histone methylation regulation in neurodegenerative disorders. Int J Mol Sci 2021;22(09):46544681.

38 Lubin FD, Sweatt JD. The IkappaB kinase regulates chromatin structure during reconsolidation of conditioned fear memories. Neuron 2007;55(06):942-957

39 Maddox SA, Schafe GE. Epigenetic alterations in the lateral amygdala are required for reconsolidation of a Pavlovian fear memory. Learn Mem 2011;18(09):579-593

40 Maddox SA, Watts CS, Schafe GE. p300/CBP histone acetyltransferase activity is required for newly acquired and reactivated fear memories in the lateral amygdala. Learn Mem 2013;20(02): 109-119

41 Stafford JM, Raybuck JD, Ryabinin AE, Lattal KM. Increasing histone acetylation in the hippocampus-infralimbic network enhances fear extinction. Biol Psychiatry 2012;72(01):25-33

42 Webb WM, Sanchez RG, Perez G, et al. Dynamic association of epigenetic H3K4me3 and DNA 5hmC marks in the dorsal hippocampus and anterior cingulate cortex following reactivation of a fear memory. Neurobiol Learn Mem 2017;142(pt. A):66-78

43 Takei S, Morinobu S, Yamamoto S, Fuchikami M, Matsumoto T, Yamawaki S. Enhanced hippocampal BDNF/TrkB signaling in response to fear conditioning in an animal model of posttraumatic stress disorder. J Psychiatr Res 2011;45(04):460-468

44 Lee JB, Wei J, Liu W, Cheng J, Feng J, Yan Z. Histone deacetylase 6 gates the synaptic action of acute stress in prefrontal cortex. J Physiol 2012;590(07):1535-1546

45 Blouin AM, Sillivan SE, Joseph NF, Miller CA. The potential of epigenetics in stress-enhanced fear learning models of PTSD. Learn Mem 2016;23(10):576-586

46 Jin B, Li Y, Robertson KD. DNA methylation: superior or subordinate in the epigenetic hierarchy? Genes Cancer 2011;2(06): 607-617

47 Khare T, Pai S, Koncevicius K, et al. 5-hmC in the brain is abundant in synaptic genes and shows differences at the exon-intron boundary. Nat Struct Mol Biol 2012;19(10):1037-1043

48 Mehta D, Bruenig D, Carrillo-Roa T, et al. Genomewide DNA methylation analysis in combat veterans reveals a novel locus for PTSD. Acta Psychiatr Scand 2017;136(05):493-505

49 Logue MW, Amstadter AB, Baker DG, et al. The Psychiatric Genomics Consortium Posttraumatic Stress Disorder Workgroup: posttraumatic stress disorder enters the age of large-scale genomic collaboration. Neuropsychopharmacology 2015;40(10): 2287-2297

50 Almli LM, Stevens JS, Smith AK, et al. A genome-wide identified risk variant for PTSD is a methylation quantitative trait locus and confers decreased cortical activation to fearful faces. Am J Med Genet B Neuropsychiatr Genet 2015;8:327-336 
51 Xie P, Kranzler HR, Yang C, Zhao H, Farrer LA, Gelernter J. Genomewide association study identifies new susceptibility loci for posttraumatic stress disorder. Biol Psychiatry 2013;74(09): 656-663

52 Duncan LE, Ratanatharathorn A, Aiello AE, et al. Largest GWAS of $\operatorname{PTSD}(\mathrm{N}=20070)$ yields genetic overlap with schizophrenia and sex differences in heritability. Mol Psychiatry 2018;23(03):666-673

53 Ashley-Koch AE, Garrett ME, Gibson J, et al; Veterans Affairs MidAtlantic Mental Illness Research, Education, and Clinical Center Workgroup. Genome-wide association study of posttraumatic stress disorder in a cohort of Iraq-Afghanistan era veterans. J Affect Disord 2015;184(184):225-234

54 Kilaru V, Iyer SV, Almli LM, et al. Genome-wide gene-based analysis suggests an association between neuroligin 1 (NLGN1) and posttraumatic stress disorder. Transl Psychiatry 2016;6(05):e820

55 Gelernter J, Sun N, Polimanti R, et al; Department of Veterans Affairs Cooperative Studies Program (\#575B) and Million Veteran Program. Genome-wide association study of post-traumatic stress disorder reexperiencing symptoms in $>165,000$ US veterans. Nat Neurosci 2019;22(09):1394-1401

56 Rutten BPF, Vermetten E, Vinkers $\mathrm{CH}$, et al. Longitudinal analyses of the DNA methylome in deployed military servicemen identify susceptibility loci for post-traumatic stress disorder. Mol Psychiatry 2018;23(05):1145-1156

57 Hammamieh R, Chakraborty N, Gautam A, et al. Whole-genome DNA methylation status associated with clinical PTSD measures of OIF/OEF veterans. Transl Psychiatry 2017;7(07):e1169

58 van Dongen J, Slagboom PE, Draisma HHM, Martin NG, Boomsma DI. The continuing value of twin studies in the omics era. Nat Rev Genet 2012;13(09):640-653

59 Zhang TY, Labonté B, Wen XL, Turecki G, Meaney MJ. Epigenetic mechanisms for the early environmental regulation of hippocampal glucocorticoid receptor gene expression in rodents and humans. Neuropsychopharmacology 2013;38(01):111-123

60 Klengel T, Mehta D, Anacker C, et al. Allele-specific FKBP5 DNA demethylation mediates gene-childhood trauma interactions. Nat Neurosci 2013;16(01):33-41

61 Li X, Wei W, Zhao QY, et al. Neocortical Tet3-mediated accumulation of 5-hydroxymethylcytosine promotes rapid behavioral adaptation. Proc Natl Acad Sci U S A 2014;111(19):7120-7125
62 Kim GS, Smith AK, Nievergelt CM, Uddin M. Neuroepigenetics of Post-Traumatic Stress Disorder. Prog Mol Biol Transl Sci 2018; 158:227-253

63 Roth TL, Zoladz PR, Sweatt JD, Diamond DM. Epigenetic modification of hippocampal Bdnf DNA in adult rats in an animal model of post-traumatic stress disorder. J Psychiatr Res 2011;45(07): 919-926

64 Gillespie CF, Bradley B, Mercer K, et al. Trauma exposure and stress-related disorders in inner city primary care patients. Gen Hosp Psychiatry 2009;31(06):505-514

65 de Quervain DJ, Aerni A, Schelling G, Roozendaal B. Glucocorticoids and the regulation of memory in health and disease. Front Neuroendocrinol 2009;30(03):358-370

66 Yehuda R, Daskalakis NP, Desarnaud F, et al. Epigenetic biomarkers as predictors and correlates of symptom improvement following psychotherapy in combat veterans with PTSD. Front Psychiatry 2013;4:118

67 Collins LJ, Schönfeld B, Chen XS. The epigenetics of non-coding RNA. In: Tollefsbol T, ed. Handbook of Epigenetics. San Diego, CA: Academic Press; 2011:49-61

68 Qureshi IA, Mehler MF. Emerging roles of non-coding RNAs in brain evolution, development, plasticity and disease. Nat Rev Neurosci 2012;13(08):528-541

69 Daskalakis NP, Rijal CM, King C, Huckins LM, Ressler KJ. Recent genetics and epigenetics approaches to PTSD. Curr Psychiatry Rep 2018;20(05):30

70 Ambeskovic M, Babenko O, Ilnytskyy Y, Kovalchuk I, Kolb B, Metz GAS. Ancestral stress alters lifetime mental health trajectories and cortical neuromorphology via epigenetic regulation. Sci Rep 2019;9(01):6389

71 Maurel OM, Torrisi SA, Barbagallo C, et al. Dysregulation of miR15a-5p, miR-497a-5p and miR-511-5p is associated with modulation of BDNF and FKBP5 in brain areas of PTSD-related susceptible and resilient mice. Int J Mol Sci 2021;22(10):5157-5177

72 Li C, Liu Y, Liu D, Jiang H, Pan F. Dynamic alterations of miR-34c expression in the hypothalamus of male rats after early adolescent traumatic stress. Neural Plast 2016;2016:5249893

73 Kang JI, Kim TY, Choi JH, So HS, Kim SJ. Allele-specific DNA methylation level of FKBP5 is associated with post-traumatic stress disorder. Psychoneuroendocrinology 2019;103:1-7 